\title{
Multi-objective optimization on mix proportions of HSHPC applied to SRC composite structures
}

\author{
Shan-Suo Zheng ${ }^{1, a}$, Lei Zeng ${ }^{1}$, Wei-Hong Zhang ${ }^{2}$, Jie Zheng ${ }^{1}$, Bin Wang ${ }^{1}$ and Lei Li $^{1}$ \\ 1 School of Civil Engineering, Xi'an University of Architecture and Technology, Xi'an, 710055, P.R. China \\ 2 Northwestern Polytechnical University, Xi'an, P.R. China
}

Received 30 March 2008, Accepted 15 Juin 2008

\begin{abstract}
The fine bond behaviour between steel shape and concrete is the base of their cooperation. For designing high strength and high performance concrete (HSHPC) applied to steel reinforced concrete (SRC) structures, a mathematical model of multi-objective and nonlinear optimization is established based on the bond-slip theory between steel shape and concrete, which takes the materials' cost and bond strength as objective functions, and takes the other performance indexes of HSHPC as constraint conditions. Optimal mix design is implemented with an application of convergent sequential quadratic programming and based on Matlab language. The optimized concrete mix proportions can satisfy all the specifications, and especially can raise the bond strength between steel shape and concrete and reduce unit cost of HSHPC.
\end{abstract}

Key words: SRC structure; HSHPC; mix proportion; optimization; multi-objective.

\section{Introduction}

Modern engineering structure advanced along the trend of long-span, heavy-load, high-rise. Attention to the durability of concrete structures promotes tremendous development in the technology of high strength and high performance concrete (HSHPC) $[1,2]$. The design of concrete mix proportions is changing from traditional strength design to multi-performance design that includes strength, durability and working performance etc. Traditional design, which depends on a method of experiential semiquantitative concrete mix proportions, will be renovated from feasibility design to optimization design. Steel reinforced high strength and high performance concrete structure is a kind of steel and concrete composite structure that applies HSHPC to steel reinforced concrete (SRC) structure. The main idea is using the good mechanical property and durable property of HSHPC to improve the cooperative performance between steel shape and concrete and increase security and durability of structures during service time [3].

This paper discusses multi-objective and nonlinear optimization design on mix proportions of HSHPC applied to SRC structures to improve synthetically performance of the structure. Design objective is transformed from traditional design of single strength target to multi-objective and nonlinear design considering working property, strength, the cooperative performance between steel shape and concrete, economic cost and endurance. For designing HSHPC applied to SRC structures, a multi-objective and nonlinear optimal mathematic model is established based on the bond-slip theory between steel shape and concrete, which takes the materials' cost and ultimate bond strength as objective functions, and takes the other performance indexes of HSHPC as constraint conditions. The optimum problem is solved by convergent sequence quadratic programming. As a result, the choice of raw material parameters and the control of construction quality will be controlled by computer automatically.

\section{Key performance indexes of HSHPC}

\subsection{Pore structure and interface transition layer}

On mesoscopic level, concrete can be regarded as composition of coarse aggregate, hardened sanded cement grout and transition region between them. Certain of concrete material's performance indexes such as strength, deformation ability, permeability and endurance are influenced by its pore structure and interface transition layer [4].

In hardened gelled material pores distribute in network shape, whose character strongly affect concrete performance. The following is a model to calculate porosity of hardened sanded cement grout, and proportion of capillary pore and gelled pore in whole pore is given as follows For capillary pore:

$$
\left[\left(W / C-\alpha\left(W / C^{*}\right)\right)\right] /\left[\left(D_{W} \times V_{c}\right)+W / C\right] .
$$

For gelled pore:

$$
\left[m g V_{g} \times \alpha\right] /\left[V_{C}+(W / C) / D_{W}\right]
$$

\footnotetext{
${ }^{a}$ Corresponding author: zhengshansuo@263.net
} 
where $W / C^{*}$ is critical water cement ratio when cement is hydrated wholly; $D_{W}$ is density of pore liquid in freshly mixed slurry; $V_{C}$ is specific volume of unhydrated cement; $V_{g}$ is average specific volume of gelatum water; $\alpha$ is percentage of hydrated cement; $m g$ is weight of gelatum water in a unit weight cement grout hydrated wholly.

According to the above model, volume of capillary pore in a unit weight cement grout is the difference between total volume and the sum volume of unhydrated cement and cement hydrate, which is denoted as $\left[W / C-\left(W / C^{*}\right) \alpha\right] D_{W}$. Volume of gelled pore in a unit weight cement grout is $m g \cdot V_{g} \cdot \alpha$.

According to the relation of water cement ratio and porosity mentioned above, it is clear that porosity of cement grout increases with increasing of water cement ratio.

Based on experiments the relation of compression strength and porosity of concrete is expressed as the following equation, which illustrates that concrete strength and Young's modulus drop along with increase of porosity.

$$
f_{c}=a(1-\rho)^{3}
$$

where $f_{c}$ is compression strength of concrete; $\rho$ is porosity; $a$ is sanded cement grout strength when porosity equals to zero.

The relation of permeation ratio and pore structure can be expressed by an empirical equation as follows

$$
K=1.684 r_{\infty}^{3.284} \times 10^{-22}
$$

where $K$ denotes permeation ratio; $r_{\infty}$ denotes maximum continuous radius, which is defined by the point of greatest gradient on mercury intrusion experiment curve.

The relation of permeation ratio, porosity and hydraulic radius is as follows

$$
\log K=38.45+4.08 \log \left(\varepsilon r_{a}^{2}\right)
$$

where $\varepsilon$ denotes porosity; $r_{a}$ denotes hydraulic radius.

Mchta and Mamohan adopted four pore parameters to establish relation with permeation ratio

$$
K=\exp \left(3.84 V_{1}+0.2 V_{2}+0.56 T D+8.09 M T P-2.53\right)
$$

where $V_{1}$ is volume ratio of pore greater than $1320 \AA$ and total pore; $V_{2}$ is volume ratio of pore between $290 \AA$ to $1320 \AA$ and total pore; TD is critical aperture, $M T P$ is ratio of total volume and hydrating degree.

\subsection{Simplified model and method to improve concrete strength}

Concrete can be simplified as composition of hardened sanded cement grout and coarse aggregate, which link together through bonding effect. The simplified model is shown in Figure 1. When bearing external force, the two portions work together and have the same stress. Because Young's modulus of them is not equal, the two portion's

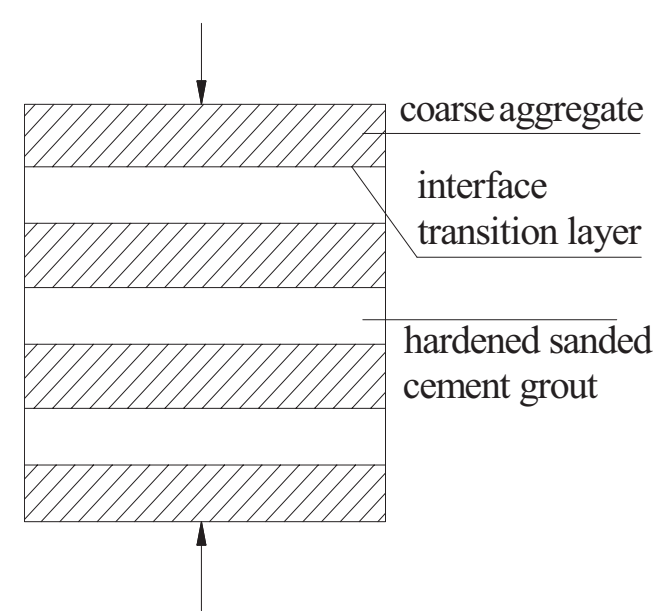

Fig. 1. Simplified concrete model.

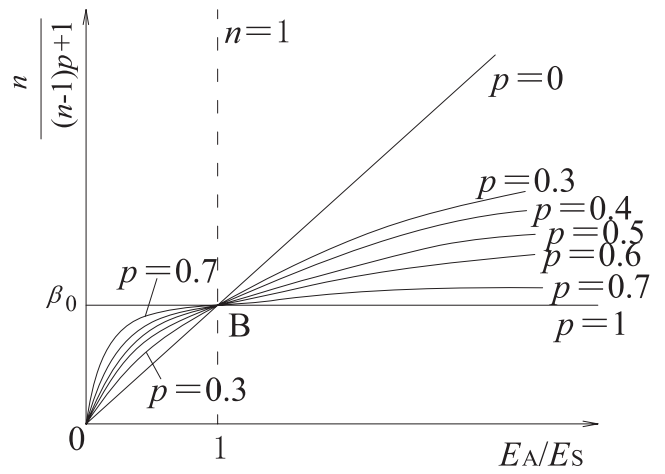

Fig. 2. Young's modulus ratio of concrete, aggregate and grout.

strain is not the same, total strain is sum of the two portions, i.e.

$$
\left\{\begin{array}{l}
\frac{R_{c}}{E_{c}}=\frac{R_{A}}{E_{A}}(1-P)+\frac{R_{S}}{E_{S}} \cdot P \\
R_{C}=R_{A}=R_{S} .
\end{array}\right.
$$

Defining $n=\frac{E_{A}}{E_{S}}$, then Young's modulus of concrete is given by

$$
E_{C}=\frac{n E_{s}}{(n-1) p+1}
$$

Defining $\beta=\frac{n}{(n-1) p+1}$, we can write

$$
E_{C}=\beta E_{S}
$$

where $E_{C}, E_{S}, E_{A}$ denote Young's modulus of concrete, coarse aggregate and hardened sanded cement grout respectively; $P$ denotes grout's volume content; $n$ denotes Young's modulus ratio of aggregate and grout; $R_{C}, R_{S}$, $R_{A}$ denote concrete's total stress, aggregate's stress and grout's stress respectively.

The above analysis indicates that cement grout and aggregate both affect concrete strength. Equations (7)-(9) and Figure 2 reflect the relationship of $P$ and $n$. In Figure 2, area $\Delta_{\alpha_{0} o B}$ represents high strength concrete, in which cement grout's strength is 
higher than aggregate. The following are methods to improve concrete strength.

\section{a. To improve $P$}

In equations (7)-(9), $n$ is definitive after materials to produce concrete are picked up. Within $\Delta_{\alpha_{0} o B}$ area shown in Figure 2, raising cement quantity can improve $P$. On the other hand, the improvement is not so effective. When $n$ equals to $0.5, P$ increases to 0.6 from 0.3 , accordingly $\beta$ increases to 0.701 from 0.588 , and cement quantity is doubled but strength is raised only $17 \%$, which means materials waste. This method is infeasible in actual engineering.

\section{b. To improve $n$}

Improving $n$ means improvement of aggregate strength. In like manner, when $P=0.5, n$ increases to 0.4 from 0.2 , accordingly $\alpha$ increases to 0.571 from 0.333 , and improvement degree is $71.5 \%$, which means applying high strength and high quality aggregate is the key point in producing high strength and high performance concrete.

\section{c. To improve $E_{S}$}

With regard to high strength and high performance concrete, coarse aggregate strength is lower than cement grout. Concrete damage lies on aggregate strength, when cement grout strength increases to a certain extent concrete strength will not be improved any more.

\subsection{Bond strength between steel and concrete}

In SRC structures there exists bonding action between steel shape and concrete, which are two kinds of materials with different performance. Bonding action enables stress to transfer effectively each other on the interface of steel and concrete and establishes working stress needed to fulfill loading capacity of structures in steel and concrete [5]. Bond stress is a kind of shear force from macroscopic effect, the experiments showed that the distribution of bond stress on the interface of steel and concrete.

Based on the theory of mechanics of materials, the distribution of bond stress along steel surface is given as follows

$$
\tau(x)=\frac{A_{s}}{u} \frac{d \sigma_{x}}{d x}=\frac{A_{s}}{u} E_{s} \frac{d \varepsilon_{x}}{d x}=\frac{-k_{1} A_{s} \varepsilon_{\max }}{u} E_{s} e^{-k_{1} x}
$$

where $E_{s}$ is elastic modulus of steel; $d \sigma_{x} / d x, d \varepsilon_{x} / d x$ are steel cross-section normal stress and normal strain increment along anchorage length respectively; $\sigma_{x}, A_{s}$ and $u$, for full section, are average stress, area and perimeter of steel section respectively, for flange or web, are average stress, area, perimeter of flange or web section respectively.

The distribution of slip between the interface of steel and concrete is given as follows

$$
s(x)=s_{\max } e^{-k_{2} x}
$$

where $s(x)$ is the slip quantity at different part of crosssection along anchorage length; $s_{\max }$ is the maximum slip quantity along anchorage length; $k_{2}$ is characteristic exponent of slip distribution.

The bond-slip pull-out test of SRC members indicates that on the interface of steel and concrete the relationship of bond strength and concrete cube compression strength is as follows $[6,7]$ :

$$
\tau_{u}=0.0130 f_{c u}+1.2150 C_{a} / h_{a}-0.0640 l_{a} / h_{a}+1.8682
$$

where $\tau_{u}$ is ultimate bond strength; $f_{c u}$ is concrete cube compression strength; $C_{a}$ is thickness of covering layer; $h_{a}$ is sectional height; $l_{a}$ is the embedded depth of steel shape.

\section{Multi-objective optimization design of concrete mix proportions}

\subsection{Mathematical model}

Mix proportion optimization design of HSHPC applied to SRC structures is a nonlinear multi-objective problem. In order to establish the relationship of every objective function and constraint conditions and finish optimum solution, multi-objective optimization technique is applied. The mathematical model of multi-objective optimization for concrete mix proportions is shown as follows [8]:

$$
\left\{\begin{array}{l}
\min \boldsymbol{F}(\boldsymbol{x}), \\
\min \boldsymbol{G}(\boldsymbol{x}) \\
\text { s.t. } \\
\boldsymbol{s}_{i}(\boldsymbol{x}) \leqslant 0, \quad i=1,2 \ldots, m \\
\quad \boldsymbol{h}_{j}(\boldsymbol{x})=0, \quad j=1,2 \ldots, l(l<n) \\
\quad \boldsymbol{x}_{i} \leqslant \boldsymbol{x} \leqslant \boldsymbol{x}_{u}
\end{array}\right.
$$

where $\boldsymbol{x}=\left[x_{1}, x_{2}, \ldots, x_{n}\right]^{T}$ is decision-making vector including $n$ components; $\boldsymbol{F}(\boldsymbol{x}), \boldsymbol{G}(\boldsymbol{x})$ is objective function vector; $\boldsymbol{s}_{i}(\boldsymbol{x}) \leqslant 0, \boldsymbol{h}_{j}(\boldsymbol{x})=0$ is constraint conditions. The decision-making Vector satisfying all restraint conditions is called feasible solution or feasible point, and the aggregate of all feasible points is called feasible aggregate. All optimization solution procedure is to find one point $\boldsymbol{x}^{*}$ to make objective function obtain extremum in this point. $\boldsymbol{x}^{*}$ is the optimal solution to the problem of concrete mix proportions accordingly.

\subsection{Constraint conditions}

In this paper concrete raw material is composed of cement, sand, macadam, mineral admixture, high efficiency water reducing agent and water, which are calling six components of concrete. Mix proportion design is to determine the consumption of each component, the consumption of these six kinds of components are expressed as $x_{1}, x_{2}, \ldots x_{6}$ individually [9]. 
a. The limits of each component consumption

$$
x_{i l} \leqslant x_{i} \leqslant x_{i u}, i=1,2, \ldots, 6
$$

where $x_{i l}, x_{i u}$ are lowest limit and maximal limit for $x_{i}$ respetively.

b. The restriction of water gel ratio

$$
k_{l} \leqslant x_{6} /\left(x_{1}+x_{4}\right) \leqslant k_{u}
$$

where, $x_{6} /\left(x_{1}+x_{4}\right)$ is the ratio of water and gel material (the sum of cement and mineral admixture); $k_{l}, k_{u}$ are lowest limit and maximal limit for water gel ratio respetively.

c. Sand ratio

$$
S_{l} \leqslant x_{2} /\left(x_{2}+x_{3}\right) \leqslant S_{u}
$$

where, $x_{2} /\left(x_{2}+x_{3}\right)$ is the ratio of sand and coarse aggregate; $S_{l}, S_{u}$ are lowest limit and maximal limit for Sand ratio respetively.

d. The total consumption of gel material

$$
C_{l} \leqslant x_{1}+x_{4} \leqslant C_{u} .
$$

e. Additive consumption

$$
R_{l} \leqslant x_{5} /\left(x_{1}+x_{4}\right) \leqslant R_{u}
$$

where, $x_{5} /\left(x_{1}+x_{4}\right)$ is the ratio of water reducing agent blend dosage and gel material dosage, and $R_{l}, R_{u}$ are its lowest limit and maximal limit respetively.

f. The restraint of material volume

Assumed that the volume of concrete equals the sum of the absolute volume of each component and the volume of air contained in concrete, the amount of raw materials in every cube concrete requires to satisfy the following equation $[10]$

$$
\sum_{i=1}^{6} x_{i} / \rho_{i}+10 \alpha-1000=0
$$

where, $\rho_{i}$ is the density of $i$ th component; $\alpha$ is the percentage of air in concrete and it equals 1 when air entraining agent not be used.

\section{g. Cube compression strength}

Admixture activity index was introduced to the calculation of mix proportions. Experimental investigation and the results of theoretical analysis show that the relationship of water gel ratio and concrete formulation strength presented by reference [11] possesses definite physical concept and better applicability, and can be applied to this optimum design. The relationship of water gel ratio and concrete formulation strength is as follows:

$$
\begin{array}{r}
A \cdot\left(-0.4952+\frac{5.514 \times x_{6}}{x_{1}+x_{4}}\right) \cdot \alpha_{a} \cdot f_{c e} \cdot\left(\frac{x_{1}+x_{4}}{x_{6}}-\alpha_{b}\right) \\
-f_{c u, k}+1.645 \sigma \geqslant 0
\end{array}
$$

where $A$ is mineral admixture activity index; $f_{c u, k}$ is concrete cube compression strength; $f_{c e}$ is cement strength; $\sigma$ is standard deviation of concrete strength; $\alpha_{a}, \alpha_{b}$ are regression coefficients.

\subsection{Objective functions}

The cooperating working ability of concrete and steel shape and the economic cost of concrete are chosen as the target of mix proportion optimization.

$\tau_{u}$, which is ultimate bond strength on the interface of steel shape and concrete, characters the cooperating working ability of concrete and steel shape. According to formula (3) the objective function of ultimate bond strength can be established as follows

$$
\tau_{u}=\sum_{i=1}^{6} F_{i}\left(x_{i}\right) x_{i}
$$

where, $x_{i}$ is the material factor of cement, aggregates, mineral admixture, etc.; $F_{i}\left(x_{i}\right)$ is effect of material factor on ultimate bond strength, and the function relationship can be defined by equations (12) and (20).

The unit price of each material component are expressed as $y_{i}$, the objective function of unit cost of concrete is as follows

$$
c=\sum_{i=1}^{6} y_{i} x_{i}
$$

\subsection{Solving process}

The mix proportion optimization problem of HSHPC applied to SRC structures can be solved by using the method of sequential quadratic programming. It is the best means to solve the optimization problem with nonlinear objective function and nonlinear constrain function. Under the support of computer calculation speed, the means constructs quadratic programming sub-problem at every iteration point, and uses the solution of this sub-problem as iterative search direction to approach the solution of constrain optimization problem [12].

Typical inequality constrained optimization question is shown as

$$
\begin{aligned}
& \min c_{0}(\boldsymbol{x}) \\
& \text { s.t. } c_{i}(\boldsymbol{x}) \leqslant 0, \quad i \in I=1,2, \ldots, m
\end{aligned}
$$

where $c_{0}(\boldsymbol{x})$ is objective function; $\boldsymbol{x}$ is design variables; $c_{i}(\boldsymbol{x})$ is constraint function of state variables. 
The following notation and definition are introduced for the current iterative point $\boldsymbol{x}^{(k)} \in \boldsymbol{R}^{n}$

$$
\begin{aligned}
& \left.\begin{array}{l}
\boldsymbol{I}_{k}^{-}=\boldsymbol{I}^{-}\left(\boldsymbol{x}^{(k)}\right)=\left\{i \in \boldsymbol{I} \mid c_{i}\left(\boldsymbol{x}^{(k)}\right) \leqslant 0\right\} \\
\boldsymbol{I}_{k}^{+}=\boldsymbol{I}^{+}\left(\boldsymbol{x}^{(k)}\right)=\left\{i \in \boldsymbol{I} \mid c_{i}\left(\boldsymbol{x}^{(k)}\right)>0\right\}
\end{array}\right\} \\
& \varphi_{k}=\varphi\left(\boldsymbol{x}^{(k)}\right)=\max \left\{0, c_{i}\left(\boldsymbol{x}^{(k)}\right) \mid i \in \boldsymbol{I}\right\} \\
& =\max \left\{0, c_{i}\left(\boldsymbol{x}^{(k)}\right) \mid i \in \boldsymbol{I}_{k}^{+}\right\} \\
& D_{i}\left(\boldsymbol{x}^{(k)}\right)= \begin{cases}c_{i}\left(\boldsymbol{x}^{(k)}\right)^{2}, & i \in \boldsymbol{I}_{k}^{-} \\
\left(\varphi\left(\boldsymbol{x}^{(k)}\right)-c_{i}\left(\boldsymbol{x}^{(k)}\right)\right)^{2}, & i \in \boldsymbol{I}_{k}^{+}\end{cases} \\
& \boldsymbol{I}\left(\boldsymbol{x}^{(k)}\right)=\left\{i \in \boldsymbol{I} \mid D_{i}\left(\boldsymbol{x}^{(k)}\right)=0\right\} .
\end{aligned}
$$

Accordingly the inequality constrained optimization can be translated into quadratic programming question.

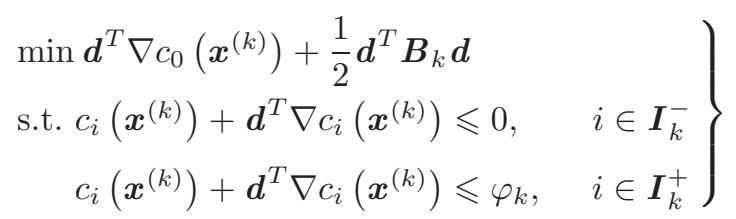

where matrix $\boldsymbol{B}_{k}$ produces convergent descending direction, and $\boldsymbol{d}$ is principal searching direction, then the solution to question (28) will approach the solution to question (23).

Quadratic programming question has its feasible solution $\boldsymbol{d}=\mathbf{0}$, assuming $\left(\boldsymbol{d}_{0}^{(k)}, \lambda^{(k+1)}\right)$ is its optimal solution.

Giving the following definition

$$
\left.\begin{array}{c}
\boldsymbol{A}_{k}=\boldsymbol{A}\left(\boldsymbol{x}^{(k)}\right)=\left(\nabla c_{i}\left(\boldsymbol{x}^{(k)}\right), i \in \boldsymbol{I}\right) \\
\boldsymbol{D}_{k}=\boldsymbol{D}\left(\boldsymbol{x}^{(k)}\right)=\left(\operatorname{diag}\left(\boldsymbol{D}_{i}\left(\boldsymbol{x}^{(k)}\right)\right), i \in \boldsymbol{I}\right)
\end{array}\right\}
$$

where $\boldsymbol{E}$ is identity matrix.

Constraint index set of quadratic programming question is $\boldsymbol{L}(k)$, and

$$
\begin{gathered}
\boldsymbol{L}_{k}=\boldsymbol{L}\left(\boldsymbol{x}^{(k)}\right)=\boldsymbol{L}_{k}^{+} \cup \boldsymbol{L}_{k}^{-} \\
\boldsymbol{L}_{k}^{-}=\boldsymbol{L}^{-}\left(\boldsymbol{x}^{(k)}\right) \\
=\left\{i \in \boldsymbol{I}_{k}^{-} \mid c_{i}\left(\boldsymbol{x}^{(k)}\right)+\left[\nabla c_{i}\left(\boldsymbol{x}^{(k)}\right)\right]^{T} \boldsymbol{d}_{0}^{(k)}\right\}
\end{gathered}
$$

$$
\begin{aligned}
\boldsymbol{L}_{k}^{+} & =\boldsymbol{L}^{+}\left(\boldsymbol{x}^{(k)}\right) \\
& =\left\{i \in \boldsymbol{I}_{k}^{+} \mid c_{i}\left(\boldsymbol{x}^{(k)}\right)+\left[\nabla c_{i}\left(\boldsymbol{x}^{(k)}\right)\right]^{T} \boldsymbol{d}_{0}^{(k)}\right. \\
& \left.=\varphi\left(\boldsymbol{x}^{(k)}\right)\right\}
\end{aligned}
$$

Quadratic programming question (28) can be generally solved in proper neighborhood of solution of inequality constrained optimization question (23), but the latter does not always have finite solution because $\boldsymbol{B}_{k}$ is not limited positive definite form, it is necessary to introduce a convergent subsidiary searching direction $q^{(k)}[13-15]$.

$$
\begin{gathered}
q^{(k)}=\rho_{k}^{\theta}\left[-\boldsymbol{P}_{k} \nabla c_{0}\left(\boldsymbol{x}^{(k)}\right)+\boldsymbol{Q}_{k}^{T} v^{(k)}\right], \theta>0 \\
\rho_{k}=\rho\left(\boldsymbol{x}^{(k)}\right)=\frac{\left\|\boldsymbol{P}_{k} \nabla c_{0}\left(\boldsymbol{x}^{(k)}\right)\right\|^{2}+\omega_{k}+\varphi\left(\boldsymbol{x}^{(k)}\right)}{1+\left|e^{T} \pi^{(k)}\right|} \\
v_{j}^{(k)}=\left\{\begin{array}{l}
-1-\rho_{k}, \pi_{j}^{(k)}<0 \\
D_{j}\left(\boldsymbol{x}^{(k)}\right)-\rho_{k}, \pi_{j}^{(k)} \geqslant 0
\end{array}\right\} \\
\pi^{(k)}=\left(\pi_{j}^{(k)}, j \in \boldsymbol{I}\right)=-\boldsymbol{Q}_{k} \nabla c_{0}\left(\boldsymbol{x}^{(k)}\right) \\
\omega_{k}=\omega\left(\boldsymbol{x}^{(k)}\right)=\sum_{j \in I} \max \left\{-\pi_{j}^{(k)}, \pi_{j}^{(k)} D_{j}\left(\boldsymbol{x}^{(k)}\right)\right\}
\end{gathered}
$$

Because matrix $\boldsymbol{A}_{k}^{T} \boldsymbol{A}_{k}+\boldsymbol{D}_{k}$ is positive definite form, the mentioned definition is of significance. If and only $\rho_{k}=0, \boldsymbol{x}^{(k)}$ is solution to optimization question (23). If $\left(\boldsymbol{d}_{0}^{(k)}, \varphi_{k}\right)=(\mathbf{0}, 0)$, then $\left(\boldsymbol{x}^{(k)}, \lambda^{(k+1)}\right)$ is solution to quadratic programming question (28).

\section{Example of mix proportion design}

Design HSHPC applied to the SRC concrete structures. The strength grade range is from C60 to C100. Preferred cement is Chinese Qingling brand P.O 52.5R normal silicate cement. Preferred coarse aggregate is from Jingyang city, Shaanxi province, China; the particle size is $5 \sim 20 \mathrm{~mm}$, and the density is $3.2 \mathrm{~kg} / \mathrm{m}^{3}$. Fine aggregate is coarse river sand from $\mathrm{Ba}$ river in Shaanxi province of China; the modulus of fineness is 2.8 , and the density is $2.61 \mathrm{~g} / \mathrm{m}^{3}$. Admixture chooses polycarboxylate high efficiency water reducing agent, and the test of cement paste fluidity shows that it is better consistency with Qingling brand cement. Choosing fly ash and silicon ash as mineral additive, which are superfine particle and own high activity. Mixing water is drinking water.

In addition, Pull-out test is made to determine/compare ultimate bond strength of optimized 


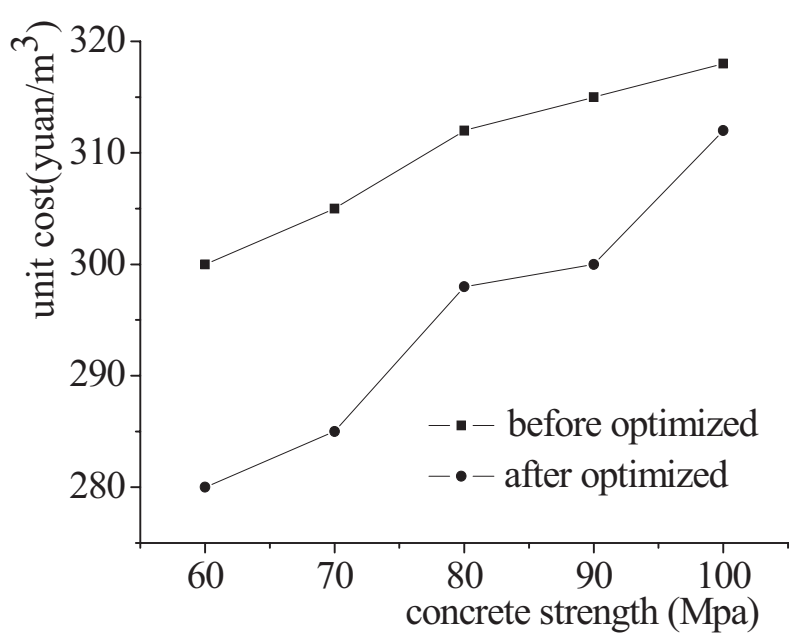

Fig. 3. Comparative analysis of concrete unit cost.

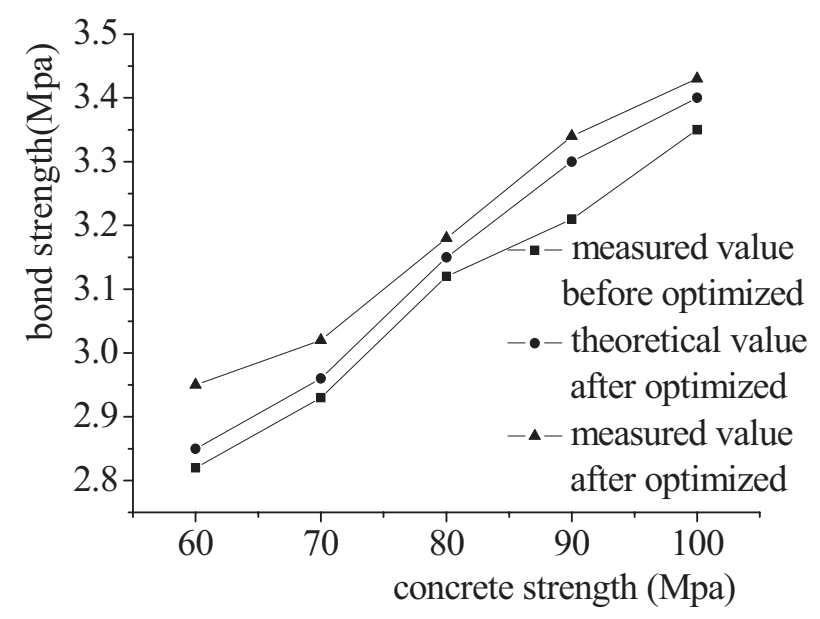

Fig. 4. Comparative analysis of ultimate bond strength.

HSHPC and/with normal HSHPC (with steel shape). I-shaped cross-section steel in the specimens is composed of two number 10 channel and $6 \mathrm{~mm}$ thick steel plate. The thickness of covering layer is $60 \mathrm{~mm}$, the anchorage length is $740 \mathrm{~mm}$.

The optimum problem is solved by convergent sequence quadratic programming and the design program is compiled based on Matlab language [16,17].

The comparison analysis of concrete unit cost and ultimate bond strength of optimized HSHPC with normal HSHPC is shown in Figures 3 and 4, and the test results of ultimate bond strength are given in Figure 4. It can be seen that the bond strength of optimized HSHPC and steel shape increases about $12 \%$, and the results of theoretical analysis with experiment is approximately consistent; cost per cubic meter can be saved about $10 \%$ through optimization.

\section{Conclusions}

HSHPC mix proportion design discussed in this paper is a kind of method of multi-objective and nonlinear optimization. Using convergent sequence quadratic programming and based on Matlab language, the optimum problem can be solved satisfactorily. Optimized HSHPC can meet all performance requirements of SRC structures and have an obvious economic benefit.

\section{Acknowledgements}

The authors would like to thank National Natural Science Foundation of China and the Educational Office of Shaan'xi Province in China for their support throughout this research.

\section{References}

1. P.K. Mehta, Greening of the concrete industry for sustainable Development, Concrete International (2000)

2. Feng Naiqian, High performance concrete structure (Beijing: Metallurgical Industry Press, 2004)

3. A. Ghezal, K.H. Khayat, J. ACI Materials 99, 264 (2002)

4. O. Kontani, S.P. Shah, Pore pressure in sealed concrete at sustained high temperatures. Proceeding Concrete Under Severe Conditions: Environment and Loading (Tokyo, Japan, 1995)

5. C.W. Roeder, R. Chmielowski, J. Struct. Eng. 125, 142 (1999)

6. S.S. Zheng, G.Z. Deng, Y. Yang, M.H. Yu, Eng. Mech. 20, 63 (2003)

7. Zheng Shansuo, Study on the bond performance between steel shape and concrete in SRC structures, Proc. of 7 th International Symposium on Structural Engineering for Young Experts (2002)

8. Zhang Wei, Xu JIaqing, Optimization Method (Shengyang: Northeast University Press)

9. J.P. Cannon, G.R. Krishna Murti, Cement and Concrete Research 1, 353 (1971)

10. Narita Takeshi, Sekino Kazuo, Concrete Research and Technology 14, 43 (2003)

11. Wan Chaojun, Concrete 41 (2002)

12. Jian Jinbao, J. Acta Math. Sci. 21, 268 (2001)

13. Chao Weihua, Guo Zheng, Optimization technique and implementation by Matlab (Beijing: Chemical Industry Press)

14. Xu Jinming, Practical tutorial of Matlab (Beijing: Tinghua University Press, 2005)

15. Xu Chengxian et al. Modern method of optimization. (Beijing: Science Press, 2002)

16. R.H. Byrd, J. Nocedal, J. Numer. Anal. 26727 (1989)

17. A. Ghezal, K.H. Khayat, Optimizing self-consolidating concrete with limestone filler by using statistical factorial design methods. ACI Mate (2002) 